\title{
El evidencial con humor entra. Acerca de su uso en los monólogos humorísticos
}

\author{
NO HUMOR, NO EVIDENTIAL: ABOUT ITS USE IN HUMOROUS MONOLOGUES
}

\section{Leonor Ruiz Gurillo}

Universidad de Alicante. Grupo GRIALE. Grupo Val.Es.Co.

Resumen

Este artículo analiza la función de los evidenciales en los monólogos humorísticos y, más concretamente, en los de Andreu Buenafuente producidos entre 2007 y 2011 (203 monólogos). Partiendo de una concepción funcional y semántica de la evidencialidad, se proponen las siguientes hipótesis: el género determina las funciones pragmáticas de la evidencialidad; los evidenciales se integran en la estructura expositivaargumentativa del monólogo; teniendo en cuenta su papel estructural, se pueden distinguir diversas estrategias de evidencialidad: y los evidenciales actúan como marcas humorísticas. Se presenta un análisis tanto cuantitativo como cualitativo de los elementos según, según dicen, dicen que, se ve que y claro. Asimismo, se propone un marco semántico-pragmático de los evidenciales y la evidencialidad en los monólogos humorísticos de Buenafuente.

PalABRAS CLAVE: humor, evidenciales, monólogo humorístico, marca humorística, ironía.
Abstract:

This paper has as its aim to shed light on functions of evidentials in humorous monologues, more precisely those by the comedian Andreu Buenafuente from 2007 to 2011 (203 monologues). Considering a functional and semantic conception of evidentiality, these hypotheses will be proposed: the genre determines the pragmatic functions of evidentiality; evidentials are integrated into the monologue's expositiveargumentative structure; regarding this structural role, several strategies of evidentiality may be distinguished; evidentials act as humorous markers. A quantitative as well as a qualitative analysis of elements según, según dicen, dicen que [it's said], se ve que [it. it is seen that'/apparently], and claro [of course] will be proposed. Furthermore, a semantic-pragmatic frame of evidentials and evidentialty in Buenafuente's humorous monologue will be explained.

KEY WORDS: humor, evidentials, humorous monologue, humorous marker, irony.

\section{INTRODUCCIÓN}

En este artículo analizamos el uso que de los evidenciales y de los diversos procedimientos de evidencialidad se llevan a cabo en un género humorístico, el monólogo. En concreto,

\footnotetext{
Este artículo ha sido posible gracias a los Proyectos de Investigación PROMETEO/2016/052 "Humor de género: observatorio de la identidad de mujeres y hombres a través del humor" (Generalitat Valenciana, 2016-19) y FFI2015-64540C2-1-P "Género, humor e identidad: desarrollo, consolidación y aplicabilidad de mecanismos lingüísticos en español" (MINECO-FEDER, 2016-19).
} 
nos apoyamos en los 203 monólogos humorísticos de Andreu Buenafuente que fueron publicados entre 2009 y 2011 integrados en los títulos Sigo diciendo (SD) (2009), Digo yo (DY) (2010) y Hablar es gratis (HG) (2011). Dichos guiones forman parte de la larga trayectoria que el cómico Andreu Buenafuente ha desarrollado como monologuista en la televisión, donde ha liderado un programa diario entre 2005 y $2015^{1}$. Estos programas del late night comenzaban con un monólogo que presenta ciertas características idiosincrásicas que serán consideradas en el análisis de los evidenciales.

Partimos de la idea de que la evidencialidad en español es un hecho semántico y funcional (Cornillie, 2007; Hassler, 2010; Albelda, 2016) en el que se desarrollan determinadas estrategias (Aikhenvald y Dixon, 2003). Con esta concepción se analizarán determinadas formas de evidencialidad que resultan más frecuentes en el corpus, como según, según dicen, dicen que, se ve que y claro. Como punto de partida se proponen las siguientes hipótesis:

(1) El género puede determinar las funciones pragmáticas de los evidenciales (Estellés y Albelda $2014,57)$. Como indican las autoras, determinadas tradiciones discursivas, como es el caso del debate parlamentario en España, facilitan que el hablante diluya su responsabilidad usando determinados evidenciales como al parecer o por lo visto. Como veremos, en los monólogos humorísticos se usan determinados evidenciales tanto para mostrar la fuente de información (directa, indirecta, etc.) como para persuadir a la audiencia.

(2) Los evidenciales se integran en la estructura expositivo-argumentativa del monólogo. Como veremos, los monólogos de Buenafuente parten con frecuencia de una noticia de actualidad y se revelan más argumentativos que narrativos. La intención básica que presentan es la de generar una opinión o persuadir a la audiencia sobre un determinado estado de cosas. En cuanto a su estructura, estos monólogos presentan una tesis, un cuerpo argumentativo y una conclusión.

(3) Pueden diferenciarse ciertas estrategias de evidencialidad, de acuerdo con su lugar estructural. Teniendo en cuenta el dominio de la evidencialidad comúnmente aceptado (Willet, 1988; Plungian, 2001; Dendale y Tasmowski, 2001), los evidenciales indirectos reproducidos (según, según dicen, dicen que, se ve que) se usan en la tesis del monólogo, mientras que los evidenciales atestiguados directos (claro) se integran en el cuerpo argumentativo del monólogo.

(4) Los evidenciales actúan como marcas humorísticas. Una marca humorística es un elemento que ayuda a interpretar el humor, mientras que los indicadores humorísticos son elementos de por sí humorísticos en un contexto dado (Ruiz Gurillo, 2014). De hecho, algunos de los evidenciales que se integran en el cuerpo argumentativo del monólogo colaboran en presentar los ganchos o los remates de los mismos ${ }^{2}$. Así, se ve que podría usarse no solo como evidencial reproducido indirecto sino también como evidencial indirecto inferido; en este último caso se manifiesta como una marca humorística de evidencialidad que guía las inferencias. Por lo que se refiere a claro, además de sus funciones epistémicas como evidencial directo, puede desarrollar funciones irónico-negativas como marca humorística de evidencialidad.

La profundización en dichas hipótesis, así como su corroboración, nos permitirá, de igual modo, reflexionar acerca de dos aspectos: por un lado, el hecho de la convencionalización (o no) de los significados evidenciales presentes en las formas evidenciales que se analizarán; por otro, las funciones contextuales que adquieren en los textos humorísticos,

\footnotetext{
${ }^{1}$ Entre enero de 2005 y junio de 2007 el programa se emitía en Antena 3 y se llamaba Buenafuente, nombre que se abreviaba como BF. Entre septiembre de 2007 y junio de 2011 se emitió en el canal LaSexta; aunque mantuvo el nombre de Buenafuente, la abreviatura cambió a BNF. Entre 2013 y 2015, Andreu Buenafuente lideró otro programa en LaSexta lamado En el aire. Con anterioridad a su etapa en la television nacional, el cómico presentó los programas La cosa nostra y Una altra cosa en la television catalana en el canal TV3. Los monólogos analizados en esta investigación pertenenen a la segunda etapa de BNF, es decir, a los monólogos que se produjeron y se emitieron entre septiembre de 2007 y junio de 2011.

${ }^{2}$ Los ganchos o jab lines aparecen en cualquier lugar del texto, mientras que el remate o punch line cierra el texto humorístico (Attardo, 2001).
} 
relacionadas con su papel como marcas humorísticas de evidencialidad que guían los significados irónico-negativos, la polifonía o la persuasión, entre otros valores.

Con el objeto de comprobar empíricamente lo hasta aquí propuesto, ilustraremos la inserción de los evidenciales en uno de los monólogos, Seguridad Social (SD, 2010: 57); a continuación, presentaremos un análisis cuantitativo de su uso en el libro del que se ha extraído dicho monólogo, Sigo diciendo (SD) (2009) (§ 2). Seguidamente se analizarán de manera separada los evidenciales con según (§ 3), dicen que (§ 4), se ve que (§ 5) y claro (§ 6). Todo ello nos permitirá establecer un marco semántico-pragmático de los evidenciales y de la evidencialidad en los monólogos humorísticos (§ 7). Las conclusiones cerrarán este trabajo (§ 8).

\section{LOS EVIDENCIALES EN LA ESTRUCTURA DE UN MONÓLOGO DE BUENAFUENTE}

Como ya se propuso en Ruiz Gurillo (2013), la estructura de los monólogos de Buenfauente vienen determinados por lo general por una noticia de la que se parte, de modo que el monólogo es principalmente expositivo-argumentativo, en lugar de narrativo, tal y como ocurre en otros monólogos humorísticos. Estos textos expositivo-argumentativos presentan una tesis que habitualmente contiene la noticia que sirve para la construcción del monólogo, a la que le sigue el cuerpo argumentativo, frecuentemente compuesto por 4 o 5 argumentos que se apoyan en ejemplos, en analogías o en la autoridad. La argumentación termina con la conclusión del monólogo donde se resumen la idea o las ideas defendidas. Por lo común, cada uno de estos argumentos o partes comienza con una premisa seria a la que le sigue una conclusión humorística. Esta conclusión humorística contiene el gancho (o jabline) o, en el caso de que se encuentre en la conclusión final, contiene el remate (o punchline) con el que se cierra el monólogo (Attardo, 2001; RuizGurillo, 2012). Así se representa en la tabla 1:

\begin{tabular}{|l|l|l|}
\hline $\begin{array}{l}\text { PARTES } \\
\text { DEL MONÓLOGO }\end{array}$ & PREMISA SERIA & CONCLUSIÓN HUMORÍSTICA \\
\hline TESIS & Premisa seria & $\begin{array}{l}\text { Conclusión humorística } \\
\text { Gancho (jab line) }\end{array}$ \\
\hline $\begin{array}{l}\text { CUERPO } \\
\text { ARGUMENTATIVO }\end{array}$ & Premisa seria & $\begin{array}{l}\text { Conclusión humorística } \\
\text { Gancho (jab line) }\end{array}$ \\
\cline { 2 - 3 } & Premisa seria & $\begin{array}{l}\text { Conclusión humorística } \\
\text { Gancho (jab line) }\end{array}$ \\
\cline { 2 - 3 } & $\ldots$ & $\ldots$ \\
\hline CONCLUSIÓN & Premisa seria & $\begin{array}{l}\text { Conclusión humorística } \\
\text { Remate (punch line) }\end{array}$ \\
\hline
\end{tabular}

Tabla 1. Estructura general de un monólogo de Andreu Buenafuente

Si consideramos esta estructura general y tenemos en cuenta la segunda hipótesis propuesta, habremos de suponer que los evidenciales se integran en dicha estructura. Como se observa en la tabla 2, el evidencial indirecto inferido se ve que se integra en la tesis del monólogo Seguridad Social (SD, 57), mientras que claro presenta dos apariciones con dos usos diferenciados en el cuerpo argumentativo: 


\begin{tabular}{|c|c|c|c|}
\hline PARTES & $\begin{array}{l}\text { ARGUMEN } \\
\text { TOS }\end{array}$ & PREMISA SERIA & CONCLUSIÓN HUMORÍSTICA \\
\hline TESIS & & $\begin{array}{l}\text { Obama quiere copiar nuestro sistema } \\
\text { sanitario. Pero, tranquilos todo tiene } \\
\text { una explicación. }\end{array}$ & $\begin{array}{l}\text { Se ve que cuando le dijeron la edad de } \\
\text { Fraga pensó que la sanidad española } \\
\text { tenía que ser la hostia. }\end{array}$ \\
\hline \multirow[t]{2}{*}{$\begin{array}{l}\text { CUERPO } \\
\text { ARGUMENTATIVO }\end{array}$} & $\begin{array}{l}1 . \\
\text { SISTEMA DE } \\
\text { DIGITALIZACI } \\
\text { ÓN }\end{array}$ & $\begin{array}{l}\text { Lo primero que hará Obama será } \\
\text { copiar el Sistema de digitalización } \\
\text { del expediente médico. Que aquí } \\
\text { estamos muy avanzados. Está todo } \\
\text { informatizado. }\end{array}$ & $\begin{array}{l}\text { De hecho, el otro día entré en el Facebook } \\
\text { y ponía: "la gripe te ha añadido como } \\
\text { amigo" }\end{array}$ \\
\hline & $\begin{array}{l}\text { 2. } \\
\text { CENTROS DE } \\
\text { ATENCIÓN } \\
\text { PRIMARIA }\end{array}$ & $\begin{array}{l}\text { Pero sobre todo lo que quieren } \\
\text { implantar allí son los centros de } \\
\text { atención primaria. }\end{array}$ & $\begin{array}{l}\text { Claro, si aquí funcionan de maravilla. } \\
\text { ¿¿Listas de espera?? ¡Qué va! Sin ir más } \\
\text { lejos, yo mañana tengo hora con el médico } \\
\text { de cabecera. A ver si me corta el cordón } \\
\text { umbilical. }\end{array}$ \\
\hline
\end{tabular}

4.

RECEPCIONI Luego están las recepcionistas que STAS te dan la hora.
Simpatiquísimas todas. Son gente que sufre mucho, eso es verdad y claro, el carácter les ha desaparecido, ya no tienen carácter.
En cualquier caso, quiero decirle al señor Obama, que sé que se está leyendo esto, que gracias por fijarse en nuestra Seguridad Social.
Pero, sobre todo, recuerde que: "Este monólogo no es de un medicamento. Ría atentamente todos los chistes. En caso de no entenderlos, consulte con Wyoming."

Tabla 2: Estructura del monólogo Seguridad Social (SD, 57).

Como vemos en este monólogo, los evidenciales se integran en diversos lugares. En la tesis aparece el evidencial indirecto se ve que para introducir la conclusión humorística de este primer argumento. El cuerpo argumentativo está compuesto por diversos argumentos. En dos de ellos claro tiene un importante papel, aunque sus funciones son diferentes. En el primer caso, claro funciona como marcador humorístico que introduce el argumento irónico (Ruiz Gurillo 2010b). En el segundo funciona con su significado de evidencia directa reforzando junto a "eso es verdad" al argumento "el carácter les ha desaparecido". En consecuencia, se detectan diversos usos evidenciales en este monólogo humorístico que van a permitir estructurar este artículo en torno a los tipos de evidencialidad y las funciones desempeñadas en los monólogos de Buenafuente.

Ahora bien, dicha ilustración no es suficiente para corroborar las hipótesis propuestas, en especial la hipótesis tercera. Dado que el libro Sigo diciendo (2009) contiene 68 monólogos de los 203 analizados, así como la mayoría de ocurrencias de evidencialidad encontradas, se presenta a continuación una tabla acerca de la inserción de las ocurrencias de evidencialidad en la tesis, el cuerpo argumentativo o la conclusión de las formas con según, dicen que, se ve que y claro: 


\begin{tabular}{|l|l|l|l|l|l|l|l|l|}
\hline & \multicolumn{2}{|l|}{$\begin{array}{l}\text { Según (+ forma libre: } \\
\text { según un estudio, } \\
\end{array}$} \\
& $\begin{array}{l}\text { Según una encuesta, } \\
\text { etc.) }\end{array}$ & \multicolumn{2}{l|}{ Se ve que } & \multicolumn{2}{l|}{} \\
\hline & Frecuencia & $\%$ & Frecuencia & $\%$ & Frecuencia & $\%$ & Frecuencia & $\%$ \\
\hline TESIS & 6 & $\begin{array}{l}46.16 \\
\%\end{array}$ & 3 & $23.07 \%$ & 2 & $11.76 \%$ & 2 & $4.34 \%$ \\
\hline $\begin{array}{l}\text { CUERPO } \\
\text { ARGUMENTATIVO }\end{array}$ & 7 & $53.84 \%$ & 9 & $69.23 \%$ & 14 & $82.35 \%$ & 44 & $95.65 \%$ \\
\hline CONCLUSIÓN & 0 & $0 \%$ & 1 & $7.69 \%$ & 1 & $5.88 \%$ & 0 & $0 \%$ \\
\hline & 13 & $100 \%$ & 13 & $100 \%$ & 17 & $100 \%$ & 46 & $100 \%$ \\
\hline
\end{tabular}

De este modo, aunque se observa una mayor frecuencia de claro en el cuerpo argumentativo, frente al resto de formas, no puede decirse lo mismo sobre dicen que o se ve que, ya que cuantitativamente sus ocurrencias son muy similares en la tesis y en el cuerpo argumentativo. En consecuencia, la hipótesis tercera no puede ser corroborada únicamente con este análisis cuantitativo, ya que no resulta suficiente para argumentar acerca de la aparición estructural de los evidenciales y sus funciones. Así pues, tales datos combinados con un análisis detallado de cada una de las formas evidenciales permitirá corroborar o desmentir esta hipótesis tercera.

Así, en primer lugar, observaremos el uso de formas de evidencialidad menos gramaticalizadas como las introducidas por según. Seguidamente se analizará el evidencial indirecto reproducido según dicen. A continuación, el papel de se ve que como evidencial indirecto reproducido y como evidencial indirecto inferido. Por último, claro será analizado en sus diversas funciones como evidencial directo atestiguado visual y como marca humorística de evidencialidad, tal y como se proponía en la segunda y la cuarta hipótesis.

\section{EVIDENCIALES INDIRECTOS REPRODUCIDOS CON SEGÚN}

La forma según introduce la fuente de conocimiento indirecto reproducido (Willet, 1988:57; Dendale y Tasmowski, 2001). En el corpus se combina con formas reproducidas que indican la fuente de forma libre (según ha dicho ella misma, según un estudio), o con expresiones más gramaticalizadas (según dicen). Por esta razón no es extraño encontrarla en la tesis del monólogo (46.16\%) para introducir la noticia de la que parte el monólogo:

(1)

Según el Instituto Nacional de Estadística, los españoles estamos ahorrando más que nunca (Andreu Buenafuente, "Ahorro familiar", SD, 143).

Aunque mantiene su función básica de introducir la fuente reproducida de la información, dado que se trata de un texto humorístico, puede introducir el gancho que sigue a la presentación de la noticia, como en (2), lo que le confiere al enunciado un carácter evaluativo humorístico:

(2)

Recientemente hemos sabido que "la esperanza de vida en España es de las más altas del mundo".

Somos el lugar con más ancianos por metro cuadrado después del plató de "Cine de barrio".

Según este estudio, nuestra esperanza de vida es de 81,1 años. Ese coma uno es inquietante.

¿Qué es? ¿Medio mes? (Andreu Buenafuente, "España, el país más longevo”, HG, 21).

Con funciones similares se encuentra según dicen que en (3) introduce una información complementaria a la noticia principal ("he leído que los españoles vamos al médico más de nueve veces al año") y que, en consecuencia, presenta un carácter reproducido:

(3) 
He leído que los españoles vamos al médico más de nueve veces al año.

(...)

Según dicen, también somos el cuarto país del mundo en consumo de fármacos. Siempre España

en el top de lo malo. (Andreu Buenafuente, "Ir al médico", DY, 92).

Por lo tanto, la fuente indirecta reproducida de la información puede referirse a datos muy concretos (según el Instituto Nacional de Estadística, según este estudio) o puede referirse a una fuente indirecta indeterminada (según dicen). También resulta frecuente el empleo de otros evidenciales reproducidos como dicen que, elemento que supone un grado mayor de consolidación que otros que señalan la fuente indirecta de información.

\section{EVIDENCIALES INDIRECTOS REPRODUCIDOS: DICEN QUE}

Dicen que es un evidencial indirecto reproducido (Willet, 1988:57; Plungian, 2001; Dendale y Tasmowski, 2001; Alvarado, 2016) que señala la fuente del conocimiento como hacen otras formas léxicas de carácter probablemente universal (Aikhenvald, 2004), como / guess, they say, I hear that. En esta línea el español presenta marcadores evidenciales reproducidos como dice (que), parece que, al parecer o por lo visto con un alto grado de gramaticalización. De modo similar a dizque en el español de Colombia, dicen que "is employed by speakers to manage the relationship among themselves, their audience, and their information source, using the concept of speaker stance" (Babel, 2009, 487). Tal y como ocurre con según dicen, su papel en los monólogos de Buenafuente es estructural, ya que ayuda a presentar con bastante frecuencia la fuente de la información, a través de la cual se ha conocido la noticia de la que parte el monólogo. Así se encuentra en la tesis del monólogo (23.07\%):

(4)

Los estudiantes de bachillerato no pasarán de curso su suspenden más de dos asignaturas. Dicen que así se lo tomarán más en serio. (Andreu Buenafuente, "Suspender bachillerato" (SD, 63).

Sin embargo, también puede encontrarse en el cuerpo argumentativo presentando uno de los ganchos (69.23\%) e introduciendo, por tanto, una evaluación humorística:

(5)

Otro juego típico del casino es la ruleta. Dicen que es como echarle una carrera a Fraga ${ }^{3}$ : tienes muchas posibilidades de ganar (Andreu Buenafuente, "Casinos", SD, 41).

Hasta ahora se han analizado diversas formas de evidencialidad indirecta presentes en los monólogos de Buenafuente (según..., según dicen, dicen que). Estas formas colaboran en la presentación de la información y tienen un carácter reproducido. Como muestra el corpus, además de señalar la fuente indirecta reproducida de la información, pueden funcionar contextualmente como guías de la evaluación humorística que hace el humorista de la noticia en el gancho humorístico. Sin embargo, destaca en el corpus la forma se ve que que presenta usos como reproducido y como evidencial indirecto inferidoindirect inferred evidential.

\section{EVIDENCIALES INDIRECTOS INFERIDOS: SE VE QUE}

A diferencia de las formas reproducidas analizadas hasta ahora, que presentan un uso principal básico que en ocasiones puede añadir un valor evaluativo, se ve que presenta en

\footnotetext{
${ }^{3}$ Político español que al final de su vida presentaba dificultades para caminar.
} 
el corpus dos usos diferenciados, marcados estructuralmente. Cuando se encuentra en la tesis del monólogo (11.76\%), es frecuente que introduzca la noticia, de igual modo que hacen según dicen, dicen que y otras formas similares. Sin embargo, en el cuerpo del monólogo (82.35\%) y en la conclusión (5.88\%) funciona frecuentemente como evidencial indirecto de carácter inferencial (Willet, 1988:57; Plungian, 2001; Dendale y Tasmowski, 2001). Ambos usos muestran funciones evidenciales de esta forma en español (Albelda, 2016), por lo que su ámbito es la proposición, presenta impersonalidad semántica, es una estructura verbal orientada al objeto y se observa construccionalización (Traugott y Trousdale, 2013) de la estructura, es decir, presenta fraseologización (Ruiz Gurillo, 1997, 2010c), ya que funciona como un todo con significado evidencial.

Cuando presenta la conclusión humorística de la tesis inicial es un evidencial indirecto reproducido, similar a dicen que. En (6) la noticia se introduce por medio de se ve que. En el mismo contexto podría encontrarse según el gobierno, según dicen, etc.:

(6)

Empezamos con una noticia relacionadas con el Plan Bolonia. Se ve que el Gobierno quiere que la informática deje de ser una Carrera y el próximo martes está prevista una manifestación de informáticos (Andreu Buenafuente, "Informáticos", SD, 61).

Las funciones evidenciales de se ve que ayudan al monologuista a llevar a cabo deducciones o inferencias a partir del tema tratado en el monólogo. Por ello, cuando aparece integrado en el cuerpo argumentativo o en la conclusión del monólogo, se ve que se usa como marca humorística de evidencialidad. Así, en (7) se inserta en un pasaje evaluativo acerca del miedo que causa Drácula. Se ve que sirve entonces para introducir el argumento incongruente, las escenas que producen más miedo que los vampiros:

\footnotetext{
(7)

Yo creo que los vampiros atraen porque a la gente, en el fondo, le gusta pasar miedo (...) En el túnel del terror, la gente paga para que la asusten. Se ve que ahora están cambiando algunas escenas; antes venía un monstruo con una sierra mecánica y ahora están poniendo a un empleado de banca con la letra de una hipoteca, que acojona más. "Uuuuuuuuh". (Andreu Buenafuente, "Drácula", SD, 108).
}

Esta secuencia secuencia evaluativa es introducida por yo creo que. Uno de los argumentos que se da en él es "en el túnel del terror la gente paga para que la asusten". A este argumento serio sigue el gancho que es introducido por el evidencial se ve que. De este modo, actúa como marca humorística de evidencialidad, ya que guía las inferencias del gancho.

También se ve que puede servir para introducir el remate que cierra el monólogo (5.88\%), como en (8), con las mismas funciones descritas en (7):
(8)
Lo que más me sorprende de Estados Unidos es lo fácil que resulta conseguir un arma. Las venden en los supermercados. Es muy peligroso. Bueno, no sé si se han enteraro... Se ve que el otro día un Chiquiprecio ${ }^{4}$ le disparó a otro. Un drama. (Andreu Buenafuente, "Estados Unidos", SD, 148)

Por lo tanto, se ve que presenta dos funciones diferenciadas en los monólogos de Buenafuente:

\footnotetext{
${ }^{4}$ Muñeco diseñado por una campaña publicitaria con el objetivo de hacer propaganda de los precios bajos de un supermercado. Se hizo muy famoso en España durante los años 90.
} 
-Puede introducir la tesis del monólogo, y concretamente la fuente de la noticia, como hacen otros evidenciales indirectos reproducidos (según dicen, dicen que,...).

-Puede introducir la conclusión humorística en el cuerpo argumentativo o en la conclusión del monólogo, de modo que funciona como un evidencial indirecto inferido. En estos casos contribuye a guiar las inferencias humorísticas de los ganchos o del remate que introduce, de modo que se convierte en una marca humorística de evidencialidad.

\section{EVIDENCIALES DIRECTOS: CLARO}

Como bien ha descrito la bibliografía (Martín Zorraquino y Portolés, 1999, Portolés, 1999, Martín Zorraquino, 2001), claro es un marcador discursivo de la evidencialidad directa atestiguada. Expresa modalidad epistémica, de tal modo que transmite que la suposición era esperable, puesto que se basa en presupuestos compartidos por hablante y oyente. En este sentido no es extraño que pueda funcionar como una marca de acuerdo entre hablante y oyente en el plano dialógico ${ }^{5}$. Así se revela de manera frecuente en el corpus en secuencias dialogales donde el monologuista representa varias voces. Además, claro, puede convertirse en una marca humorística que introduce el gancho humorístico y que, en consecuencia, permite obtener inferencias negativas del argumento que introduce.

Cuando claro funciona como un evidencial directo se encuentra en fragmentos de discurso directo donde funciona como marca de acuerdo. En (9) sirve para introducir el acto de respuesta afirmativa de la segunda voz que ha de representar el monologuista en el escenario y que en el texto escrito se marca con la acotación "lo hace":

(9)

Ahora los únicos que tienen dinero son las entidades de préstamos rápidos. De estos que "sí, sí, sí,... jte fiamos! "Huy, sí, cómo me fío." Tú vas a Cofidis ", que tiene nombre de supermercado, y te encuentras un mundo de felicidad. Todos son superamables y te sonrien con cara de Michael Landon en La casa de la pradera (Lo hace): hay un tío tirando dinero. "Bienvenido a Cofidis, chico. ¿Quieres dinero? Claro hombre. ¿Cuánto? ¿Treinta mil? Toma cuarenta mil y quédate con el cambio" (Andreu Buenafuente, "Bancos", DY, 23.)

Ahora bien, los usos de claro como marcador humorístico evidencial son mucho más frecuentes y propios de este género humorístico. Claro se convierte en estos casos en una marca que guía el gancho hacia inferencias irónico-negativas. Como ha propuesto el grupo GRIALE, la ironía conlleva inferencias negativas que desencadenan la inversión de los principios pragmáticos (Rodríguez Rosique, 2009, 2013; Ruiz Gurillo, 2010b). Esta explicación se corrobora igualmente en el análisis de la ironía y los evidenciales presentado por Santamaría (2009) o Gilmour et al. (2010). En tales contextos, claro, como marca de evidencia directa, se ha de inferir contextualmente como una marca que guía la ironía del contexto. De este modo, la ironía de la proposición se infiere de forma inversa o negativa. Además, estos son casos de ironía insertos en textos humorísticos, de manera que la ironía está al servicio del humor (Ruiz Gurillo, 2012, 131-141) y, consecuentemente, claro es una marca humorística de evidencialidad (negativa).

\footnotetext{
${ }^{5}$ Este es uno de los valores principales que le otorga Pons (1998:169-172) y (2006), que señala además que el acuerdo es una categoría eminentemente dialógica.

${ }^{6}$ Entidad que concede préstamos personales.
} 
La corroboración de que los usos preferentes de claro como evidencial epistémico de acuerdo se mantienen en contextos irónico-humorísticos se encuentra en ejemplos como el de (10). En este caso, el monologuista representa varias voces en un entorno claramente irónico, lo que lleva a una interpretación negativa del contexto, donde claro permite guiar dichas inferencias. Nótese que las acotaciones, que hacen referencia a los gestos que debe hacer el monologuista al representar el monólogo, ayudan a la interpretación irónica de lo dicho:

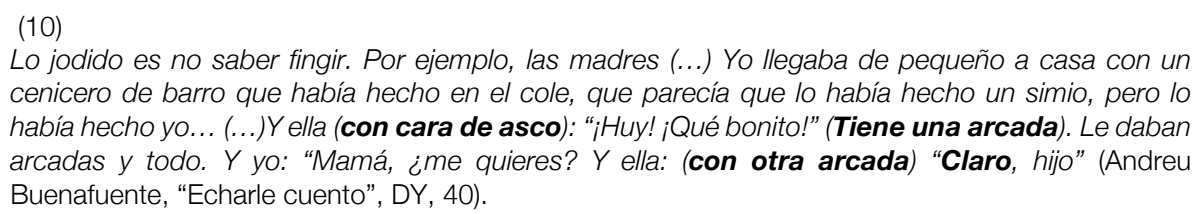

Como se ha afirmado, claro se encuentra como marca humorística de evidencialidad de modo frecuente en nuestro corpus. Así aparece en (11), donde, pese a los dos marcadores de acuerdo (sí, claro), la única interpretación posible del gancho es la inversa a lo dicho, "la gente no puede ir a esquiar" y, consecuentemente, contribuye a la argumentación de que esquiar es un deporte muy caro:

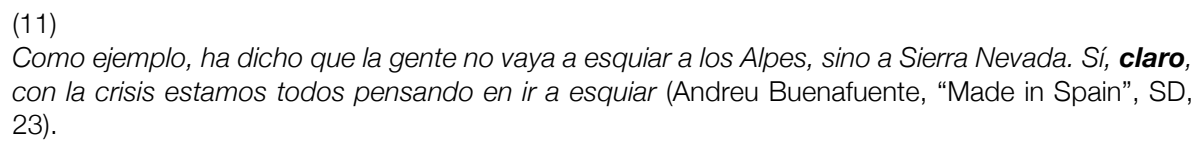

En otros ejemplos se confirma que, pese a funcionar como marca humorística, conserva su valor epistémico y muestra las presuposiciones compartidas entre Buenafuente y sus destinatarios:

(12)

Ayer, una señora de 92 años fue detenida en Barajas con cuatro kilos de cocaína encima. Pesaba más la cocaína que ella. Claro, no puedes decir que es para consumo propio. A lo mejor la señora dijo que era harina, para rebozar pechugas (Andreu Buenafuente, "Presupuestos", SD, 155-156).

Por lo tanto, claro se revela en el corpus como un marcador epistémico de evidencialidad directa. Aunque este uso convencionalizado aparece con cierta frecuencia en el corpus, resulta mucho más frecuente y prominente el empleo de claro como marca de evidencialidad irónico-humorística. En estos contextos, claro se inserta en la conclusión humorística que sigue al argumento serio y guía hacia una interpretación negativa.

\section{HACIA UN MARCO SEMÁNTICO-PRAGMÁTICO DE LOS EVIDENCIALES Y LA EVIDENCIALDIAD EN EL MONÓLOGO HUMORÍSTICO}

Hasta el momento se han analizado por separado diversos procedimientos de evidencialidad presentes en los monólogos humorísticos de Andreu Buenafuente. Es conveniente ahora reflexionar acerca del uso de dichos procedimientos de evidencialidad en estos monólogos. Como bien indica Peterson et al. (2010:4) los evidenciales suponen un reto para la sintaxis la semántica y la pragmática, hecho que también se manifiesta en el análisis presentado de estos elementos en el monólogo humorístico. Centrándonos en los tres principales temas de la pragmática de la evidencialidad según Peterson et al. (2010:4), la discusión que sigue se organizará en torno a tres preguntas:

1. ¿Cómo se usa la evidencialidad para codificar la información? 
2. ¿Cómo se usa la evidencialidad en el nivel del acto de habla?

3. ¿Cómo se usa la evidencialidad para implicar otros significados?

Así pues, se hablará a continuación de los evidenciales analizados como elementos que señalan la información evaluativa, como elementos que actúan en el nivel de los actos de habla y como elementos que implican otros significados, tales como la ironía.

Los evidenciales indica no solo la referencia a la fuente, sino que pueden codificar el predicado evaluativo. De hecho, como bien indica Mushin (2000: 928), "evidentials are tools for speakers to anchor information in particular discourse contexts not only to the source but also to their assessment of that source". Este carácter evaluativo se observa como significado contextual en algunos de los contextos analizados. Si bien es cierto que el español ha codificado algunos de sus evidenciales para expresar la fuente de la información, como al parecer o por lo visto (Ruiz Gurillo, 2004, 2006, González Ramos, 2005; Cornillie, 2007; RAE, 2010, Martín Zorraquino, 2010, Kotwica, 2013; Estellés y Albelda 2014), los analizados en este trabajo presentan grados menores de gramaticalización. Ahora bien, señalan de igual modo la fuente indirecta de la información según dicen, dicen que y se ve que. En algunos contextos, como en (2) y (5), según este estudio y dicen que pueden introducir de modo contextual la evaluación del gancho o del remate. En otros casos se ve que expresa evidencialidad inferida como una evaluación de los hechos, como en (7) y (8). Concretamente, se ve que en (7) se inserta en un pasaje evaluativo del monólogo dentro del cuerpo argumentativo, lo que favorece su empleo como elemento evaluativo que introduce el gancho del fragmento. Dentro de los procesos evaluativos de estos evidenciales, cabe señalar también su función polifónica, ya que algunos de ellos se insertan en pasajes dialogales, como claro en (9) y (10).

Los evidenciales actúan sobre el acto ilocutivo y modifican la proposición. Tanto los que se insertan en la introducción de la noticia como los que lo hacen en el cuerpo argumentativo o en la conclusión del monólogo le sirven al monologuista para indicar la fuente de información de la que parte, ya se indique de manera específica (según el Instituto Nacional de Estadística), ya de un modo genérico (según dicen, dicen que). Dado que la referencia a la fuente de la información es en sí mismo un mecanismo argumentativo considerable (Bermúdez, 2002), el uso de tales procedimientos otorga al monologuista cierto grado de autoridad acerca de lo que desarrolla en el monólogo y favorece su ethos ${ }^{7}$ como comediante (Greenbaum, 1999; Ruiz Gurillo, 2012). Una vez situado en este nivel en el que indica la fuente de la información, emplea otros elementos de evidencialidad que marcan la inferencia de ciertas informaciones (se ve que) o emplea, principalmente en el cuerpo argumentativo, el evidencial directo claro. Esos elementos sirven para guiar los ganchos o remates, de modo que adquieren una función argumentativa como elementos que colaboran en la persuasión. Concretamente, guían a la audiencia hacia determinados efectos perlocutivos propios del humor, como la risa, la parodia o la diversión (Ruiz Gurillo, 2013).

\footnotetext{
${ }^{7}$ Consideramos con Greenbaum (1999) que el stand-up Comedy americano y, por extensión, los monólogos humorísticos en español que heredan dicho sistema, se basan en la retórica para lograr su objetivo de persuadir a la audiencia. Así, el monologuista usa su ethos para desarrollar y mantener su autoridad cómica y, en consecuencia, hacerla fiable y creíble. Además emplea el kairós para adaptarse al público de cada noche; su talento natural; y la teoría y la práctica, todo lo cual le facilita conseguir los efectos que persigue con el monólogo.
} 
En cuanto a los otros significados implicados, "evidentials are used to convey another meanings such as mirativiy, metaphor and irony" (Peterson et al., 2010:3). De hecho, la ironía, que conduce al ámbito de la negación (Gilmour et al. (2010), Rodríguez Rosique, 2009, Santamaría 2009), puede usar diversas marcas de evidencialidad, entre las que destaca claro en nuestro corpus. A partir de sus empleos polifónicos, como en (10), se inserta en un contexto irónico en el que ayuda a guiar la proposición hacia inferencias negativas. El empleo de ironía en contextos como el de (11) facilita los efectos humorísticos.

Así pues, cabe argumentar que los evidenciales hallados en el corpus actúan como elementos importantes en la evaluación, la polifonía, la argumentación, la persuasión, la ironía y los efectos humorísticos perseguidos por el monologuista. Ahora bien, cabría diferenciar entre los que presentan usos más convencionalizados, propios de otros textos, como según dicen o dicen que, y los que adquieren funciones propiamente humorísticas como marcas. Tal es el caso del valor inferencial de se ve que y de los usos irónicohumorísticos de claro.

Considerando tales ideas, pueden distinguirse diversos tipos, a partir de su aparición estructural en el monólogo y de las funciones desempeñadas. En primer lugar, los evidenciales indirectos reproducidos (según dicen, dicen que) que presentan en el corpus un significado convencionalizado, presente también en otros géneros. Estos se encuentran en la presentación de la noticia o en el cuerpo argumentativo; cuando aparecen en el cuerpo de la noticia, pueden colaborar contextualmente en la evaluación humorística del gancho. En segundo lugar, el evidencial se ve que, que presenta dos funciones diferenciadas marcadas estructuralmente: una primera de carácter indirecto reproducido, y una segunda de carácter indirecto inferencial; en este segundo caso, se ve que se manifiesta frecuentemente como marca humorística de evidencialidad. Por último, el evidencial directo claro, que principalmente aparece en el cuerpo argumentativo, puede desarrollar en contextos irónico-humorísticos funciones como marca humorística de evidencialidad. La tabla 4 resume las funciones de los evidenciales en los monólogos humorísticos de Andreu Buenafuente analizados: 


\begin{tabular}{|c|c|c|c|c|c|}
\hline EVIDENTIALES & $\begin{array}{l}\text { FUNCIONES DE } \\
\text { LOS } \\
\text { EVIDENCIALES }\end{array}$ & $\begin{array}{l}\text { SIGNIFICADO } \\
\text { CONVENCIONAL }\end{array}$ & $\begin{array}{l}\text { FUNCIONES } \\
\text { PRAGMÁTICAS }\end{array}$ & $\begin{array}{l}\text { INSERCIÓN } \\
\text { PREFERRENT } \\
\text { E EN LA } \\
\text { TRAMA } \\
\text { HUMORÍSTICA }\end{array}$ & $\begin{array}{l}\text { FUNCIONES } \\
\text { HUMORÍSTICAS }\end{array}$ \\
\hline Según... & $\begin{array}{l}\text { EVIDENCIAL } \\
\text { REPRODUCIDO } \\
\text { INDIRECTO }\end{array}$ & $\begin{array}{l}\text { Fuente de } \\
\text { información }\end{array}$ & (evaluación) & Tesis & \\
\hline Según dicen & $\begin{array}{l}\text { EVIDENCIAL } \\
\text { REPRODUCIDO } \\
\text { INDIRECTO }\end{array}$ & $\begin{array}{ll}\text { Fuente } & \text { de } \\
\text { información } & \end{array}$ & & Tesis & \\
\hline Dicen que & $\begin{array}{l}\text { EVIDENCIAL } \\
\text { REPRODUCIDO } \\
\text { INDIRECTO }\end{array}$ & $\begin{array}{ll}\text { Fuente } & \text { de } \\
\text { información } & \end{array}$ & (evaluación) & $\begin{array}{l}\text { Tesis/cuerpo } \\
\text { argumentativo }\end{array}$ & \\
\hline $\begin{array}{l}\text { Se ve que } \\
\text { [lit.] }\end{array}$ & $\begin{array}{l}\text { EVIDENCIAL } \\
\text { REPRODUCIDO } \\
\text { INDIRECTO }\end{array}$ & $\begin{array}{l}\text { Fuente de } \\
\text { información }\end{array}$ & & Tesis & \\
\hline $\begin{array}{l}\text { Se ve que } \\
\text { [apparently] }\end{array}$ & $\begin{array}{l}\text { EVIDENCIAL } \\
\text { INDIRECTO } \\
\text { INFERENCIAL }\end{array}$ & $\begin{array}{l}\text { Información } \\
\text { inferida }\end{array}$ & $\begin{array}{l}\text { Evaluación } \\
\text { Argumentación }\end{array}$ & $\begin{array}{l}\text { Cuerpo } \\
\text { argumentativo }\end{array}$ & Marca humorística \\
\hline Claro & $\begin{array}{l}\text { EVIDENCIAL } \\
\text { DIRECTO } \\
\text { ATESTIGUADO } \\
\end{array}$ & $\begin{array}{l}\text { Información } \\
\text { inferida }\end{array}$ & $\begin{array}{l}\text { Epistemicidad } \\
\text { Polifonía }\end{array}$ & $\begin{array}{l}\text { Cuerpo } \\
\text { argumentativo }\end{array}$ & \\
\hline Claro & & & $\begin{array}{l}\text { Ironía-negación } \\
\text { Argumentación } \\
\text { Persuasión }\end{array}$ & $\begin{array}{l}\text { Cuerpo } \\
\text { argumentativo }\end{array}$ & Marca humorística \\
\hline
\end{tabular}

\section{CONCLUSIONES}

El análisis cuantitativo y cualitativo presentado en este trabajo permite, en consecuencia, desmentir o corroborar las hipótesis planteadas:

(1) El género humorístico, esto es, el monólogo y específicamente las características idiosincrásicas de los monólogos de Buenafuente, determinan las funciones que desarrollan en ellos los evidenciales.

(2) Estos evidenciales se integran en la estructura argumentativa propia de los monólogos de Buenafuente, descrita pormenorizadamente en Ruiz Gurillo (2013). Así, aparecen en la tesis, en el cuerpo argumentativo o en la conclusión.

(3) Sin embargo, no pueden diferenciarse diversas estrategias de evidencialidad marcadas únicamente por su integración estructural en el monólogo y su aparición en la tesis, en el cuerpo argumentativo o en la conclusión. Este aspecto se ha complementado con las funciones que desarrollan en el corpus, lo que ha permitido diferenciar diversas tendencias: los evidenciales de carácter indirecto reproducido (según, según dicen, dicen que) presentan esta función como la principal, aunque pueden colaborar contextualmente en la evaluación que introduce el gancho; el evidencial se ve que presenta dos usos diferenciados, marcados estructuralmente: el primero de ellos, con significado literal, es de carácter indirecto reproducido; el segundo, con el significado de 'aparentemente', es de carácter indirecto inferencial. Por su parte, claro muestra una preferencia de aparición en el cuerpo argumentativo (95.65\%), aunque este hecho de manera aislada no permite corroborar los usos expuestos como evidencial directo y como marca humorística. Así, hemos visto que en el cuerpo argumentativo puede desarrollar en ocasiones funciones como marca irónico-humorística de evidencialidad.

(4) Una de las estrategias de evidencialidad señaladas en este género humorístico es la de guiar las inferencias humorísticas, esto es, la de actuar como marcas humorísticas de evidencialidad. En concreto, se ve que presenta estos valores cuando es un evidencial indirecto inferencial. Por su 
parte, claro se revela en ciertas ocasiones como una marca humorística de evidencialidad que guía lo dicho hacia interpretaciones irónico-negativas.

El análisis aquí planteado de los evidenciales en los monólogos y, más concretamente, en los de Andrés Buenafuente, ha permitido establecer algunas tendencias interesantes de su empleo. Ahora bien, los datos tendrían que ser ampliados con un corpus más amplio de monólogos, así como con otros géneros humorísticos (parodias, chistes, etc.) con el fin de afianzar las hipótesis aquí defendidas.

\section{REFERENCIAS}

\subsection{Corpus:}

DY=BUENAFUENTE, Andreu (2009): Digo yo (Los monólogos de La Sexta). Barcelona: Planeta. SD=BUENAFUENTE, Andreu (2010): Sigo diciendo (Los monólogos de La Sexta). Barcelona: Planeta. HG=BuENAFUENTE, Andreu (2011): Hablar es gratis (Los monólogos de La Sexta). Barcelona: Planeta.

\subsection{Bibliografía:}

AIKHENVALD, Aleksandra (2004): Evidentiality. Oxford: University Press.

AIKHENVALD, Aleksandra. Y., and R. M. W. DIXON (eds) (2003): Studies in evidentiality. Amsterdan: John Benjamins.

AlbeldA, Marta (2015): “Evidentiality in non-evidential languages: Are there evidentials in Spanish?” Journal of Pragmatics 85: 135-137.

AlbeldA, Marta (2016): "La expresión de la evidencialidad en la construcción se ve (que)." Spanish in Context, 13:2: 237-262.

Alvarado OrTEGa, M. Belén (2016): «Enunciación y percepción: la evidencialidad en los textos turísticos del español», Onomázein, 33: 327-342.

ATTARDO, Salvatore (2001): Humorous Texts: A Semantic and Pragmatic Analysis. Berlin: Mouton de Gruyter.

BABEL, Anna M (2009): "Dizque, evidentiality, and stance in Valley Spanish". Language in Society 38: 487-511.

BERMúDEZ, Fernando (2002): “¿De dónde lo sacaste?: elevación de sujeto y gramaticalización de la evidencialidad en castellano." En García NEGRONI, M. Actas del Congreso Internacional La Argumentación. Buenos Aires, Universidad de Buenos Aires, 174-189.

BERmúdEZ, Fernando (2005): Evidencialidad. La codificación lingüística del punto de vista. Stockholm: Universitet.

CORNILLIE, Bert (2007): Evidentiality and Epistemic Modality in Spanish (Semi-)Auxiliaries. A Cognitive-Functional Approach. Berlin: Mouton de Gruyter.

DENDALE, P. y L. TASMOWSKI (2001): "Introduction: evidentiality and related notions", Journal of Pragmatics, 33, 3, 339-348.

Estellés-Arguedas, María y Marta Albelda-MARCo (2014): "Evidentials, politeness and prosody in Spanish: A corpus analysis." Journal of Politeness Research 10(1): 29-62.

GILMOUR, Drew; Ashleigh GONZALES; Meagan LOUIE. (2010): "Evidentials and Parasitic Irony: Activating the Illocution-Proposition Distinction." En Tyler PERTERSON and Uli SAUERLAND, Evidence from Evidentials, The University of British Columbia Working Papers in Linguistics, 28, 1-6.

GONZÁlEZ RAMOS, Elisa (2005): "Por lo visto y al parecer: comparación de dos locuciones modales epistémicas." Interlingüística 16, 1: 541-554.

GONZÁLEZ, C (2011): "Estrategias gramaticales de expresión de la evidencialidad en español de Chile." Revista Alpha, 32, 149-165.

GREENBAUM, Andrea (1999): "Stand-up comedy as rhetorical argument: An investigation of comic culture", Humor 12-1: 33-46.

HASSLER, Gerda (2010): "Epistemic modality and evidentiality and their determination on a deictic basis. The case of Romance languages." En DIEWALD, Gabriele and Elena SMIRNOVA, The Linguistic Realization of Evidentiality in European Languages, Berlin, Mouton de Gruyter, , 223- 247.

KotwicA, Dorota (2013): "Al parecer evidencial atenuante. ¿Y reforzador?" Congreso de la Asociación de Jóvenes Lingüistas (AJL).

Martín ZorRaquino, Ma Antonia, y José Portóles (1999): "Los marcadores del discurso." Ignacio Bosque y Violeta DEMONTE (Dirs.) Gramática descriptiva de la lengua española, Madrid, Espasa, 4051-4213. 
MARTín ZorRAQUINO, María Antonia. 2010. "Las partículas discursivas en los diccionarios y los diccionarios de partículas discursivas (con referencia especial a desde luego / sin duda y por lo visto /al parecer)." . En Elisenda Bernal, Sergi TorneR, and Janet DeCesaris (ed.), Estudis de Lexicografia, Barcelona, Institut Universitari de Lingüística Aplicada. Universitat Pompeu Fabra, Documenta Universitaria, 2003-2005.

Peterson, Tyler; Rose-Marie Déchaine; Uli SAUERLAND (2010): "Evidence form Evidentials: Introduction. In Evidence from Evidentials, En Tyler PERTERSON and Uli SAUERLAND, Evidence from Evidentials, The University of British Columbia Working Papers in Linguistics, 28, 1-6.

PLunGIAN, V. A (2001): "The place of evidentiality within the universal grammatical space." Journal of Pragmatics, 33, 3. 415-438.

Pons, Salvador (1998): Conexión y conectores. Estudio de su relación en el registro informal de la lengua. València: Universitat.

Pons, Salvador (2006): "Claro 1 y 2." Diccionario de partículas discursivas del español. http://www.dpde.es.

PORTOLÉs, José (1998): Marcadores del discurso. Barcelona: Ariel Practicum.

RAE (2010): Nueva gramática la lengua Española, II. Madrid: Espasa Calpe.

RodríGUEZ-Rosique, Susana (2009): «Una propuesta neogriceana." En Leonor RuIz-GuRILlo, and Xose A. PADILla García (eds.), Dime cómo ironizas y te diré quién eres. Una aproximación pragmática a la ironía, Frankfurt Peter Lang, 109-132.

RUIz GURILLO,, Leonor (1997): Aspectos de fraseología teórica española. València: Universitat.

RuIz GuRILlo,, Leonor (2004): "Las locuciones marcadoras del español: análisis y aplicaciones", En Ramón AlmelA, Estanislao Ramón Trives y Gerd WotJAK (eds.) Fraseología contrastiva, Murcia, Universidad, 241257.

Ruiz GuRILlo, Leonor (2006): Hechos pragmáticos del español. Alicante: Universidad de Alicante.

RuIz GuRILlo, Leonor (2010a): "Para una aproximación neogriceana a la ironía en español." Revista Española de Lingüística, 40/2: 95-124.

RuIz GURILLO,, Leonor (2010b): "Las 'marcas discursivas' de la ironía." En José Luis CIFUENTES, Antonio GómEZ, Antonio LILLO, José MATEO, and Francisco Yus (eds.), Los caminos de la lengua. Estudios en homenaje a Enrique Alcaraz Varó, Alicante, Universidad de Alicante, 871-886.

RUIz GURILLO,, Leonor (2010c): "Interrelaciones entre fraseología y gramaticalización en español." Revista de Filología Española. LX, 10: 173-194.

RUIz GURILLO,, Leonor (2012):La lingüística del humor en español. Madrid: Arco/Libros.

RUIZ GURILLO,, Leonor (2013): "Narrative strategies in Buenafuente's humorous monologues." En Leonor RuIz Gurillo y M. Belén AlvaRAdo OrTEga (eds.), Irony and Humor: From Pragmatics to Discourse, Amsterdam, John Benjamins, 107-140.

Ruiz GuRILlo,, Leonor (2014): "Infiriendo el humor. Un modelo de análisis para el español." Revista CLAC (Círculo de Lingüística Aplicada a la Comunicación), 59: 148-162.

SANTAMARíA, Isabel (2009): «Los evidenciales.» En Leonor RuIz-GuRILLO, and Xose A. PADILLA GARcía (eds.), Dime cómo ironizas y te diré quién eres. Una aproximación pragmática a la ironía, Frankfurt Peter Lang, 267-292.

TraugotT, Elizabeth C., and Graeme Trousdale (2013): Constructionalization and Constructional Changes. Oxford: Oxford University Press.

WILLET, Thomas (1988): "A cross - linguistic survey of the grammaticization of evidentiality." Studies in Language, 12. $51-97$. 